\title{
Strategies for Improved Global Electromagnetic Response Estimates
}

\author{
R. J. BANKS \\ Department of Environmental Sciences, University of Lancaster, \\ Lancaster, England
}

(Received April 28, 1981; Revised September 5, 1981)

\begin{abstract}
Efforts to make more precise and reliable measurements of the electromagnetic response of the Earth at periods less than a few days are baffled by the contamination of the magnetic variation data by currents which flow in the heterogeneous outer shell of the Earth. In this paper, quantitative estimates of the potential contamination are made by analysing induction in an Earth model which, in addition to the usual steep rise in conductivity at a depth of $500 \mathrm{~km}$, also involves a superficial conducting layer of integrated conductance $4,000 \mathrm{~S}$. It is concluded that (a) it may be possible, by careful selection of suitable stretches of data from observatories far from conductivity anomalies, to extend global electromagnetic response estimates to periods as short as 8 hours, but that (b) present response estimates in the period range 1-10 days may be contaminated by the currents in the outer shell.

These hypotheses are tested by an investigation of the spatial and temporal variations in geomagnetic transfer functions observed at stations in Europe during a three-month period. Two events recorded during that time show distinctly different responses in the period range 1-3 days. The first yields temporally and spatially consistent values of the transfer function linking vertical and horizontal north components. The excitation is most probably of ring current origin, and the bulk of the induced current flows in the deep, spherically symmetric part of the Earth. The transfer functions generated by the second event are also consistent with time, but differ substantially from one obervatory to another. The source of this event is a current system which is spatially non-uniform. The transfer function is partly determined by the configuration of the localised external current system, and partly by the geometry of induced currents in the surface sheet. Both factors give rise to persistent relationships between the magnetic field components, which contaminate the global response estimates.
\end{abstract}

\section{Introduction}

Our knowledge of the variation with depth of electrical conductivity within the Earth depends upon the availability of accurate estimates of the electromagnetic response of the Earth to magnetic field variations of external origin, and on the ability to interpret or invert these data. The last ten years has seen a steady improvement in the methods available for extracting the conductivity distribution from the response data, starting with the work of BAiley (1970), PARKer (1971), and WeIdelt (1972), and culminating in the very recent paper by PARKER (1980). 
Unfortunately, these advances on the theoretical front have not been matched by significant improvements in the quality or frequency range of the electromagnetic response data set. This is not to deny the value of those analyses which have been made, particularly of the periodic and quasi-periodic variations, such as the annual (MALIN and IsIKARA, 1976), eleven-year solar cycle (HARwOOD and MALIN, 1977, Ducruix et al., 1980) and daily variation (MALIN, 1977). Nonetheless, there seems to be a lack of confidence in the data as a whole, judging by the number of inversion attempts which restrict themselves to fitting relatively small subsets. If a satisfactory data base existed, it would be automatically required that a conductivity model should fit the whole, rather than a part of it. The rejection or replacement of one element of the data set would not necessarily invalidate the remainder.

Uncertainty as to the validity of a response estimate can take a number of different forms, but must be given quantitative expression to be of any practical use. Random errors should be made as small as possible, but they are probably the easiest with which to deal. Once they have been defined in terms of a confidence interval, they can be treated in the manner suggested by PARKER (1980). A far greater problem is the question of whether the response estimates are truly applicable to the model which will be used in their inversion. The existence of, for example, a spherically symmetric conductivity structure compatible with the response data, can be established by tests of the kind proposed by WEIDELT (1972), and these should certainly be applied. Nevertheless, a data set may meet such constraints and yet contain systematic inaccuracies.

The worst problems with which the data analyst must contend are the effects on the global response of lateral variations in the conductivity of the outer shell of the Earth, and of incorrect assumptions about the spatial uniformity or structure of the magnetic fields which induce the currents. The aim of this paper is to analyse these effects, to assess their importance, and to propose methods for minimising their influence on estimates of the global electromagnetic response.

\section{The Dependence of Induction on Frequency and Wave Number}

Most of the current induced in the Earth by magnetic field variations with periods of several hours or more flows in two distinct ranges of depth. The surface shell, which includes the oceans, shows great lateral variability in its conductivity; the structure below the conductivity 'step' at $400 \mathrm{~km}$ or more, is generally assumed to be spherically symmetric. Any current induced in the outer shell is available to be redistributed by the laterally varying conductivity, and the associated perturbations in the electromagnetic field will alter the electromagnetic response at observatories which happen to lie close to current concentrations. The magnitude of the distortion will depend in the first place on the relative proportions of the internal current which flow in the surface and deep conductors. As a guide to the size of the effect, we can compute the fraction of induced current flowing in the surface layer of a realistic conductivity structure, and investigate its dependence on the frequency and wavenumber of the inducing field.

The model chosen as the basis for the calculations is that proposed in my 1972 paper, which involves a steep rise in conductivity in the range $500-700 \mathrm{~km}$. The outer $400 \mathrm{~km}$ of 
the model has a uniform conductivity of $10^{-2} \mathrm{Sm}^{-1}$. However, a surface layer $40 \mathrm{~km}$ thick, with a uniform conductivity of $10^{-1} \mathrm{Sm}^{-1}$, has been superimposed on the original structure. For the purposes of this calculation, the thickness and conductivity are not individually important; it is the conductivity-thickness product, $4,000 \mathrm{~S}$, which matters. If the oceans were everywhere $5,000 \mathrm{~m}$ deep, their integrated conductivity would be $20,000 \mathrm{~S}$. However, the resistive continental masses obviously reduce the effectiveness of induction, though by how much is not clear. The effect will itself depend on the spatial scale of the inducing fields, being greatest for fields with spatial scales substantially larger than the horizontal dimensions of the oceans. LAHIRI and PRICE (1939) inferred the existence of an equivalent shell with a conductivity-thickness product of $4,000 \mathrm{~S}$ from their studies of $S_{q}$ and $D_{s t}$ variations, and I have retained their estimate for want of a better alternative.

The computational methods described in BANKs (1969) and PRICE (1962) were employed to compute the depth dependence of the currents induced (a) in a spherical Earth by spherical harmonic fields of degree $n$, and (b) in a plane Earth by fields of spatial wavenumber $v$. In fact, only one calculation was strictly necessary, since the two geometries can be transformed one into the other (WEIDELT 1972). The results are summarised in Fig. 1, in which the percentage of current flowing in the surface shell is plotted as a function of frequency for different spatial scales of the inducing field. The shaded areas identify the frequency ranges and spatial scales of the two main sources of geomagnetic variations: the ring current and the auroral electrojet.

The symmetric ring current gives rise to a series of odd degree zonal spherical

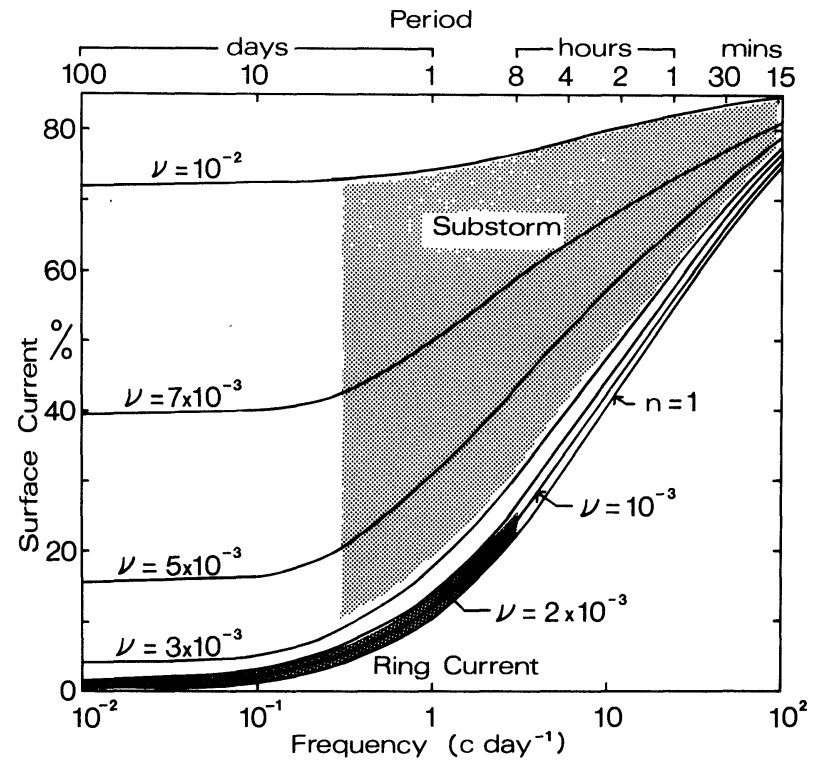

Fig. 1. Percentage of the total current induced in the Earth which flows in the surface shell, as a function of frequency and spatial wavenumber (unit of $v$ is $\mathrm{km}^{-1}$ ) or spherical harmonic order (n). The heavy and light shaded areas indicate the ranges of wavenumbers and frequencies generated by the ring current and magnetic substorms respectively. 


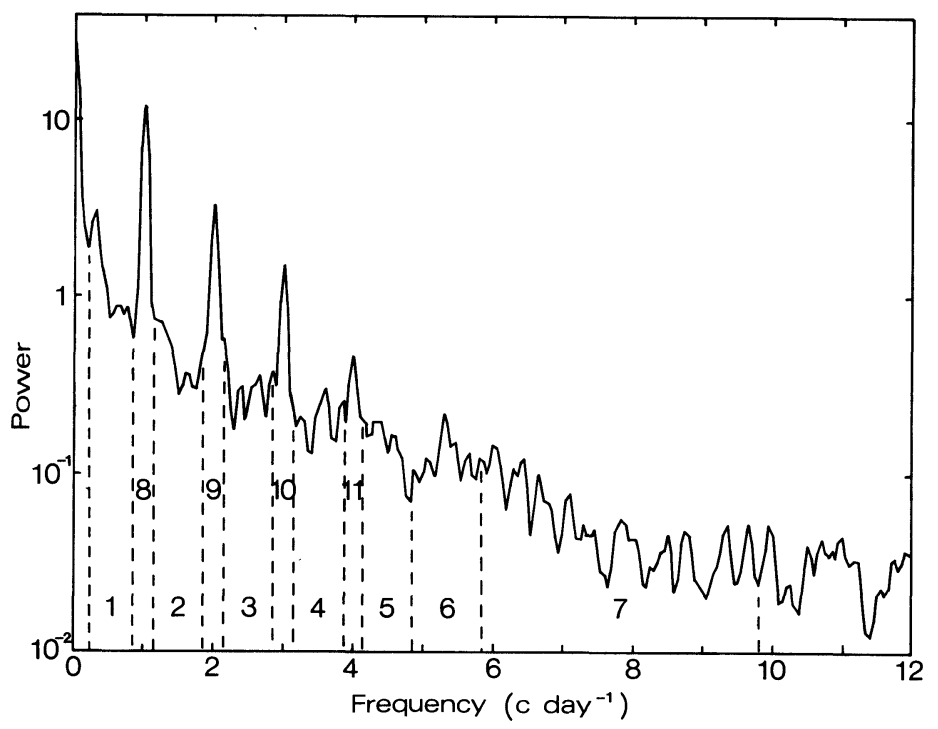

Fig. 2. Power spectrum of Stonyhurst $H$ component, showing the bands chosen for demodulation. The resolution is $0.05 \mathrm{c} \mathrm{day}^{-1}$, the unit of power $20(\mathrm{nT})^{2}$ day.

harmonics. Because of its distance from the Earth, the amplitude of the terms decreases rapidly as the degree of the harmonics increases. The size of the $P_{3}^{0}$ term in the expansion is generally less than $10 \%$ that of the $P_{1}^{0}$ term, and a similar progression is to be expected on passing to the $P_{5}^{0}$ term. The magnetic fields of the quiet daily variation $\left(S_{q}\right)$ are likewise adequately represented by low degree spherical harmonics $(n<4)$. Neither of these groups of sources is likely to generate fields with spherical harmonic components of degree greater than 6 , or spatial wavenumbers $v$ greater than $10^{-3} \mathrm{~km}^{-1}$.

The range of frequencies generated by the ring current is more difficult to establish. There can be little doubt that it is the predominant source of variations with periods greater than 5 days. What is more doubtful is the extent to which the ring current contributes to the spectrum at shorter periods, particularly less than one day. There does not seem to be any theoretical reason why its intensity should not fluctuate at frequencies higher than $1 \mathrm{c} \mathrm{day}^{-1}$. However, most of the power generated by the ring current does not originate from continuous oscillations, but from sharp bursts which occur at irregular intervals, and which form the main and recovery phases of magnetic storms. A simple model of an individual storm is a negative spike followed by a recovery of the form $\exp -\beta t$, generating a frequency spectrum proportional to $\left(\beta^{2}+\omega^{2}\right)^{-1}(\omega$ is the angular frequency). A typical decay time, $\tau=\beta^{-1}$, for the recovery phase of a storm is 1 day, which would produce a spectrum decaying to half its zero frequency value at a frequency of $1 \mathrm{c} \mathrm{day}{ }^{-1}$. The amount of ring current generated power in this portion of the spectrum will depend on the amplitude and recurrence rate of the storms, and also on the decay constant: the more rapid the recovery of the storm, the more slowly the spectrum decays. The difficulty in dealing with this part of the spectrum is not that ring current generated energy does not exist, but that it is usually swamped by other sources, which include the 
asymmetric ring current that often develops in the early stages of the main phase of storms. The $S_{q}$ variation is not subject to this limitation, since the power is concentrated into lines at well-defined frequencies. The $1,2,3$, and $4 \mathrm{c} \mathrm{day}^{-1}$ harmonics usually stand out clearly above the background, and have a substantial power advantage over it (see, for example, Fig. 2).

Substorms involve intense concentrations of ionospheric current in the auroral zones. Measurements by magnetometer arrays (HUGHES and ROSTOKER, 1979a, b) suggest that typical wavelengths of the electromagnetic fields generated by the electrojets are of the order of $1,000 \mathrm{~km}$, ranging perhaps from 2,000 to $500 \mathrm{~km}$. The corresponding range of wavenumbers is from $3 \times 10^{-3}$ to $10^{-2} \mathrm{~km}^{-1}$. Fields of larger spatial scale are associated with the return paths of the electrojets, but their amplitude is much smaller. The frequency range of the fluctuating fields generated by these spatially inhomogeneous sources probably covers much of the spectrum. However, they make their biggest contribution at periods of less than a day.

Although such arguments cannot place very precise bounds on the frequency/ wavenumber ranges generated by the different sources, the definition is good enough to show that the change from global induction to induction in the surface sheet takes place in the frequency range $10^{-1}$ to $10 \mathrm{c} \mathrm{day}^{-1}$, and is assisted by the change in spatial scales of the sources. Figure 1 demonstrates that methods of analysis of different parts of the frequency spectrum must take account of the following factors:

(i) At periods of 10 days or more, the dominant source is the symmetric ring current. The uniformity of the fields it generates, and their low frequency, together ensure that a negligible amount of current is induced in the outer shell. The measured electromagnetic response of any station should be truly representative of the spherically symmetric Earth.

(ii) At periods of 2-10 days, the dominant source is still the symmetric ring current, and, provided an observatory is not too close to a zone of current concentration, the observed response will be the global response appropriate to induction by a low order spherical harmonic. However, it may be necessary to discriminate against some data from certain observatories. Substorm activity and partial ring currents will at times contribute to this part of the spectrum, and, when they do so, will induce a significant amount of current in the outer shell. The resultant contamination will be compounded at unfortunately sited observatories.

(iii) $S_{q}$ response estimates at periods of 24 and 12 hours will not be severely contaminated at favourably located observatories, but at 6 hours or less serious contamination is likely to be the rule rather than the exception.

(iv) In the period range 4 hours-2 days (excluding $S_{q}$ ) the source fields are usually non-uniform, and a substantial proportion of the induced current flows in the outer shell. Unless stations are favourably sited, the response will be distorted to an unacceptable degree. However, there may be periods of time when there is little substorm activity, and instead disturbances originating in the symmetric ring current. Estimates of the response made during such periods would be closer to the global value.

(v) At periods less than 4 hours, whatever the source, the major part of the induced current flows in the surface layer. The response at most stations will be determined by the 
geometry of the conductive pathways and not by the vertical distribution of conductivity. At periods less than 300 seconds, a genuine local induction process may again take over, with the measured electromagnetic fields determined by the local vertical conductivity structure (CONNERNEY and KUCKES, 1980).

The two major conclusions as far as the improvement of global response estimates is concerned are:

(a) On the positive side, it may be possible to extend global response estimates to periods as short as 8 hours by choosing appropriate stretches of data at suitable observatories.

(b) On the negative side, it looks as though more care is needed in the period range 1-10 days if contamination of the response by currents in the surface shell is to be avoided.

\section{The Electromagnetic Response at Intermediate Frequencies}

The previous section attempted to estimate the amount of current potentially 'available' in the surface sheet at different frequencies. We now consider the way in which the surface currents, and also localised external currents, may affect methods of estimating the global spherically symmetric electromagnetic response. A technique often employed is to compute transfer functions relating different components of the magnetic field measured at a single observatory. If certain conditions are met, the transfer function can be directly linked to the global response. However, there are other ways in which a consistent transfer function may be generated which do not relate at all to the global response.

\subsection{The response of the outer shell}

Once current has been induced in the surface sheet, it behaves on a local scale as though it were a direct current, and is concentrated by regions where the conductivity is high, and deflected away from those where it is low. The response of a highly conducting zone in the sheet can be characterised by the anomalous electromagnetic fields which are created when it is immersed in regional currents flowing in two orthogonal directions. When a uniform regional current flows in the $x$ direction, $J_{x}$, a perturbation current is set up in and around the conductivity anomaly: $\boldsymbol{j}_{x}\left(x^{\prime}, y^{\prime}\right)\left(x^{\prime}, y^{\prime}\right.$ are coordinates centred on the anomaly. The perturbation current is a vector function of position, but the subscript refers to the regional current which excites it). Similarly, a uniform regional current in the $y$ direction, $J_{y}$, creates a perturbation current $j_{y}\left(x^{\prime}, y^{\prime}\right)$. In each case, the strength of the perturbation current is proportional to the strength of the exciting current:

$$
j_{x}\left(x^{\prime}, y^{\prime}\right)=\boldsymbol{G}_{x}\left(x^{\prime}, y^{\prime}\right) J_{x}
$$

and

$$
\boldsymbol{j}_{y}\left(x^{\prime}, y^{\prime}\right)=\boldsymbol{G}_{y}\left(x^{\prime}, y^{\prime}\right) J_{y} \text {. }
$$

The direction of the perturbation current, on the other hand, is controlled by the geometry of the conductor and the position of the observer, through the vector functions $\boldsymbol{G}_{x}$ and $\boldsymbol{G}_{y^{\prime}}$ and is independent of the strength of the regional current. The perturbation 
current produced by an arbitrary regional flow is the vector sum of the terms in Eq. (1):

$$
\boldsymbol{j}\left(x^{\prime}, y^{\prime}\right)=\boldsymbol{G}_{x}\left(x^{\prime}, y^{\prime}\right) J_{x}+\boldsymbol{G}_{y}\left(x^{\prime}, y^{\prime}\right) J_{y} .
$$

It is monitored most directly by means of the vertical component $Z$, of the magnetic field. If the part of the vertical field which originates from the perturbation currents can be isolated, it can be transformed directly into an equivalent current stream function in the sheet (BANKS, 1979). The regional current flow is monitored by means of the horizontal components $H$ and $D$ of the magnetic field. For them to truly represent the regional current, they should be measured at some distance from the conductivity anomaly. However, the anomalous horizontal field is usually small in comparison with the regional field, and the values of $H$ and $D$ which are used are often those observed at the same site where $Z$ is measured. Within the limits of validity of this approximation, Eq. (2) can be transformed into a relationship between the components of the magnetic field recorded at a single station:

$$
Z\left(x^{\prime}, y^{\prime}\right)=A\left(x^{\prime}, y^{\prime}\right) H\left(x^{\prime}, y^{\prime}\right)+B\left(x^{\prime}, y^{\prime}\right) D\left(x^{\prime}, y^{\prime}\right) .
$$

This equation is the familiar transfer function relationship utilised in so-called magnetic deep sounding experiments, first established on an empirical basis by PARKINSON (1958) and others. At mid-latitude stations, in the period range 10 minutes- 3 hours, it is usually possible to fit at least $70 \%$ of the total $Z$ component power by such a relationship.

It follows from this discussion that the transfer or electromagnetic response functions, $A$ and $B$, at a particular station, may tell us little or nothing about the absolute variation of electrical conductivity with depth. Instead, they are determined by the geometry of those conductivity anomalies which are sufficiently close for their perturbation currents to produce a detectable anomalous part of the vertical component of the magnetic field.

\subsection{The global response}

The symmetric ring current generates a field with a spatial structure which can be approximated by a single, $P_{1}^{0}$, spherical harmonic. If the induced current flows only in the spherically symmetric part of the Earth, the vertical and horizontal components of the magnetic field at a station whose co-latitude is $\theta$ are

$$
\left.\begin{array}{l}
Z(\theta, \omega)=\left(e_{1}^{0}(\omega)-2 i_{1}^{0}(\omega)\right) \cos \theta \\
H(\theta, \omega)=-\left(e_{1}^{0}(\omega)+i_{1}^{0}(\omega)\right) \sin \theta \\
D(\theta, \omega)=0
\end{array}\right\}
$$

$\left(i_{1}^{0}(\omega)\right.$ and $e_{1}^{0}(\omega)$ are respectively the internal and external parts of the $P_{1}^{0}$ potential at frequency $\omega$ ). Equation (4) can be rewritten as a transfer function relationship between the vertical and horizontal components of the magnetic field at the observatory:

$$
Z(\theta, \omega)=\left\{\frac{1-2 Q_{1}^{0}(\omega)}{1+Q_{1}^{0}(\omega)}\right\}\left\{-\frac{\cos \theta}{\sin \theta}\right\} H(\theta, \omega)
$$

or 


$$
\begin{aligned}
& Z(\theta, \omega)=G(\theta, \omega) H(\theta, \omega) \\
& \left(Q_{1}^{0}(\omega)=i_{1}^{0}(\omega) / e_{1}^{0}(\omega)\right) .
\end{aligned}
$$

In this case, the single-station transfer function, $G(\theta, \omega)$, contains the required information about the radial distribution of conductivity in the spherically symmetric part of the Earth.

\subsection{The response at high magnetic latitudes}

The two types of response analysed in the previous sections correspond to two extreme models of the electromagnetic induction process. They make quite different predictions about the information contained in the relationships observed between magnetic field components at a single station. In the first case, the mechanism of induction is ignored, and it is assumed that all of the vertical field which correlates with the horizontal field originates in internal currents within the surface sheet. In the second case, it is assumed that the correlated vertical field originates from a combination of external and internal currents, which produce well defined, persistent, and spatially uniform electromagnetic fields.

However, persistent correlations between the vertical and horizontal components of the field can also be generated by much less uniform sources, the most important of which is the auroral electrojet. A simple model of the electrojet consists of an east-west line current at a height $h$ above the Earth's surface. The induction effects can be represented by an image current flowing at depth $(h+d)$ beneath a perfect conductor located at a depth $d$. A source with this geometry clearly produces correlated $Z$ and $H$ magnetic field variations. The $Z / H$ response initially varies linearly with horizontal distance from the source $(x)$, reaching a maximum value at $x=(h(h+2 d))^{1 / 2}$, and thereafter decreasing approximately as $x^{-1}$. If $h$ and $d$ are assumed to be 100 and $400 \mathrm{~km}$ respectively, the critical distance is $300 \mathrm{~km}$, and since most of the observatories of interest lie further south of the auroral zone than this, their electrojet response should show the $x^{-1}$ fall-off.

At geomagnetic latitudes less than $50^{\circ}$, the contribution made by vertical fields originating directly from the electrojet is probably small. The remaining 'normal' part of the vertical field will be associated with ionospheric and magnetospheric return currents of a much less localised and directional character. Over a period of time, there should be no persistent correlation between the components of such fields, and the assumption that they can be treated as an uncorrelated residual term in a transfer function analysis is probably justified.

At magnetic latitudes greater than $50^{\circ}$, such an assumption is more dubious. The vertical field originating directly from the electrojet will be a more significant fraction of the total. Its relation to the horizontal component will not be random. Although the $Z / H$ line current response will vary, depending on the position of the auroral oval relative to the observatory, it will do so only between certain limits. Even when an average is taken over a great deal of data, a correlation may remain between the vertical and horizontal components, which will be related to the observatory's mean position relative to the electrojet, and to the depth of the currents which it induces. 


\subsection{The effect on response estimates}

A common analytical procedure, used in forming estimates of the global electromagnetic response, is to compute the spectra of long stretches of hourly or daily mean values of the vertical and horizontal components of the magnetic field recorded at an observatory, and then to fit a relationship of the form

$$
Z(\omega)=\tilde{G}(\omega) H(\omega)+\varepsilon(\omega)
$$

to the Fourier Transforms by minimising the mean square misfit $\left\langle\varepsilon(\omega) \varepsilon^{*}(\omega)\right\rangle$. A possible estimator of the response function would be

$$
\widetilde{G}(\omega)=\frac{\left\langle Z(\omega) H^{*}(\omega)\right\rangle}{\left\langle H(\omega) H^{*}(\omega)\right\rangle}
$$

where the brackets represent averages over adjacent frequency estimates, or different stretches of data. We would hope that $\tilde{G}(\omega)$ is an unbiased estimate of the true global $P_{1}^{0}$ response, $G(\omega)$, defined by Eq. (5). Averaging over longer stretches of data should lead to more reliable values of $\tilde{G}(\omega)$, with smaller standard errors.

The assumption which underlies such expectations is that there is a single stable relationship between $Z(\omega)$ and $H(\omega)$ throughout the length of the data series, and that it is the one defined by Eq. (5), associated with induction by the ring current in a spherically symmetric Earth. However, we have seen that there are two additional mechanisms by which relatively stable relationships between the vertical and horizontal components may be generated. In the course of a single magnetic record, the source may change from ring current to substorm, with a corresponding switch from global (Subsection 3.2) to surface (Subsection 3.1) induction modes. Alternatively, an equatorward shift in the position of the auroral oval may mean that an observatory previously giving a 'clean' global $P_{1}^{0}$ response subsequently yields response estimates severely contaminated by electrojet effects (Subsection 3.3). The resultant transfer function will be an average of the response functions for the different modes of induction, but weighted according to the level of horizontal field power generated by each source. Using more mixed-mode data will not lead to a reduction in the error estimates, nor will it be possible to interpret the transfer function in terms of any single induction model.

If the magnetic variations can be examined in the time domain, before they are combined to form power and cross spectra, it may be possible to distinguish the events which give the purest global response, and to reject those which are badly contaminated by surface effects. The technique most suited to a mixed frequency/time domain analysis is complex demodulation, which generates 'time-local' estimates of the amplitude and phase of the disturbance in the frequency bands of interest (BANKS, 1975). The demodulated time series can then be used to form time-local estimates of the transfer functions, which should reveal whether the hypothesized 'mode mixing' really occurs.

\section{European Observatory Responses at Intermediate Frequencies}

\subsection{Complex demodulation}

The first step in complex demodulation is to decide how best to split the spectrum 
into distinct frequency bands. The choice must be made on the basis of (a) the source mechanism, (b) the power levels, and (c) the information content of the resulting response estimate in relation to the conductivity structure.

Figure 2 shows the power spectrum of the $H$ component recorded at Stonyhurst. The raw data consisted of hourly mean values for the period October 1st-December 31st, 1964. The daily variation and its harmonics at 2,3 , and $4 \mathrm{c}$ day ${ }^{-1}$ are clearly recognisable as lines in the spectrum, with widths determined by the characteristics of the spectral window. Beyond $7 \mathrm{c} \mathrm{day}^{-1}$, the spectrum flattens out, suggesting that the level of the digitisation noise has been reached. At frequencies less than $7 \mathrm{c} \mathrm{day}^{-1}$, the background decays roughly exponentially with increasing frequency. In this case, the division of the spectrum is determined by the need to isolate the $S_{q}$ lines, with their distinct source mechanism, from the background. Bands 8-11 have been chosen for this purpose, with fairly generous widths. Bands 1-4 fill the gaps between the lines; the source may be ring current or substorm. Finally, three further continuum bands, 5-7, have been picked, with increasing widths to compensate for the decreasing power levels.

Each of the bands was demodulated in turn, and the modulus of the resulting complex time series plotted, to show the variation of the level of activity throughout the period of the record. Figure 3 shows the modulus of the $H$ component demodulates for band 1 (period range 1-3 days, centred on 2 days) for a number of European observatories, including Stonyhurst. They span a range of latitude of approximately $10^{\circ}$, from Eskdalemuir in the north to Roburent in the South. The 'waveform' of the demodulates is very consistent at all the observatories, although there are differences in amplitude. Between 15 and 35 days, there is a recurrent disturbance, which may represent

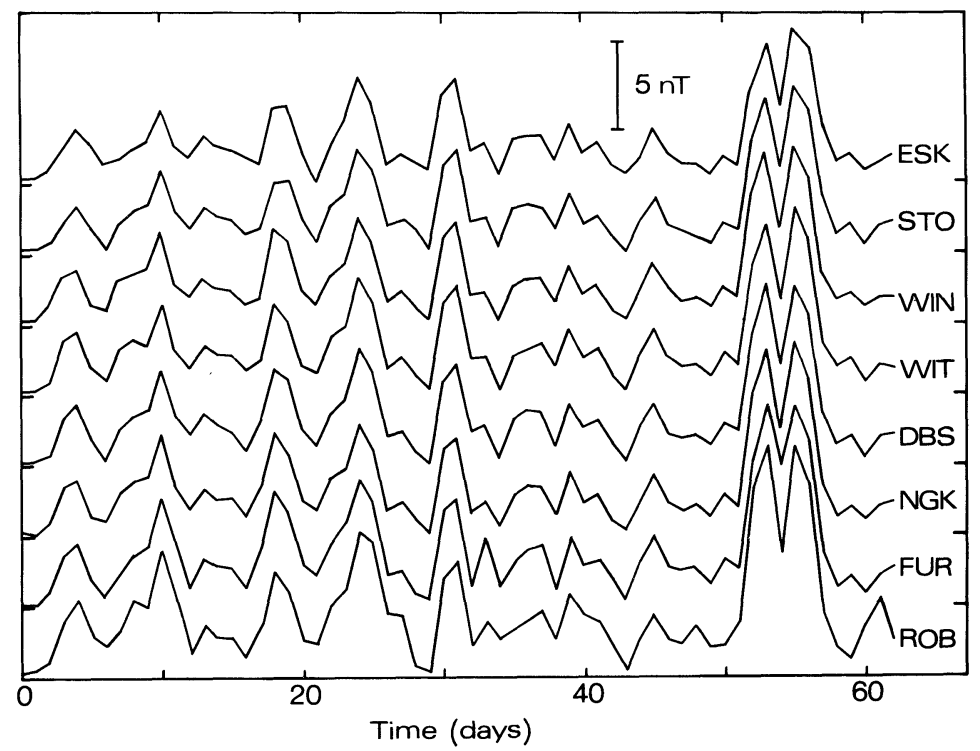

Fig. 3. Demodulates of the $H$ component at European observatories for Band 1 (periods 1-3 days). Sampling interval=1 day. (ESK, Eskdalemuir; STO, Stonyhurst; WIN, Wingst; WIT, Witteveeen; DBS, Dourbes; NGK, Niemegk; FUR, Furstenfeldbruck; ROB, Roburent) 


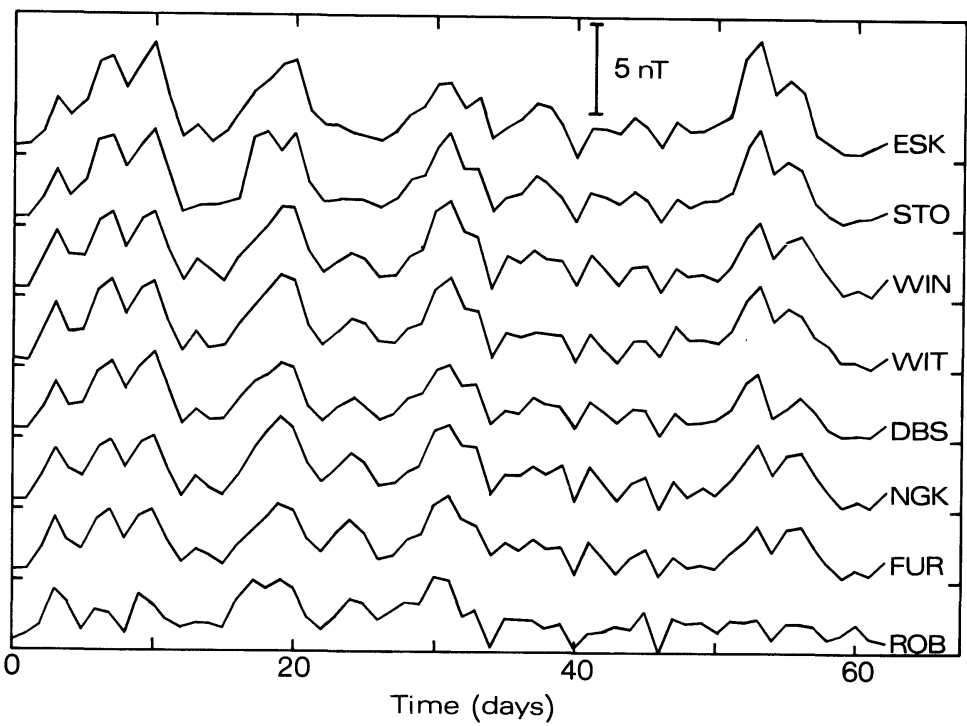

Fig. 4. Demodulates of the $D$ component.

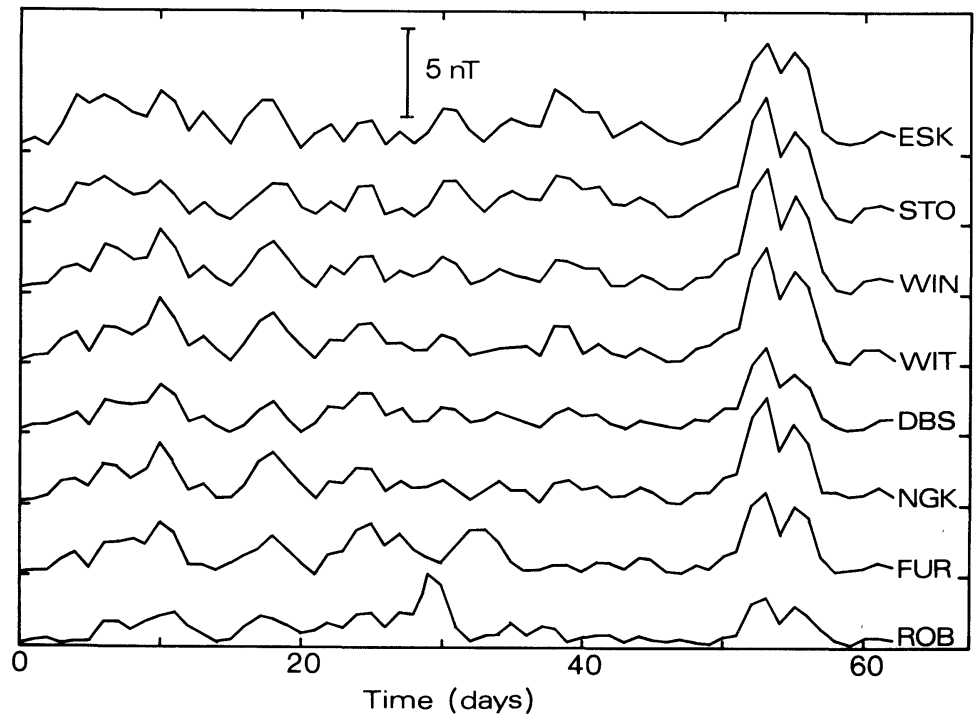

Fig. 5. Demodulates of the $Z$ component.

a series of events associated with the interaction of solar wind sector boundaries with the magnetosphere. The amplitudes are very similar at all stations during this time. The other principal event occurs between 50 and 60 days, and, although the shape of the envelope is similar, its magnitude changes significantly from north to south.

Figure 4 shows the changing levels of activity of the $D$ component. The same remarks apply, but this time the north-south variation in the 50-60 day event is more 
dramatic - it disappears almost completely at Roburent. The vertical component (Fig. 5) displays similar spatial variability. In addition, Roburent disagrees totally with the other observatories between 10 and 40 days, and there is a spike in the Furstenfeldbruck record at 30-35 days not seen elsewhere. These latter 'events' are clearly fictitious, and represent a recurring problem in long period $Z$ records, where signal power levels are relatively low.

In summary, two main groups of events can be recognised in this stretch of data. A general, moderate level of disturbance exists between 10 and 40 days, which correlates well from place to place, and is of uniform amplitude in all components at all stations. A large amplitude event occurs between 50 and 60 days, which is present in all observatory records, but which shows considerable spatial variation, particularly in $D$ and $Z$, both in amplitude and 'waveform'.

\subsection{Time-local transfer functions}

Time-local spectra and cross spectra of the magnetic field components were computed by averaging appropriate products of the demodulates within a window of length 11 days. Equation (3) was fitted to the data, and the transfer functions $A(\omega), B(\omega)$ determined from the spectra and cross-spectra of $H, D$, and $Z$ (BANKs, 1973). Because only 11 data points contribute to these response estimates, the standard errors will be correspondingly large. Furstenfeldbruck and Roburent have been omitted from the calculations because of the discrepancies in their $Z$ records.

Figure 6 shows the modulus of $A(\omega)$ for band 1 . The record can be divided into three segments: event 1 , lasting from 15 to 35 days; event 2 , from 50 to 60 days; and the rest. Outside the limits of events 1 and 2, the response estimates are very scattered: they oscillate considerably at any one observatory, as well as showing substantial differences among the observatories. There is no clear pattern, in striking contrast to the behaviour

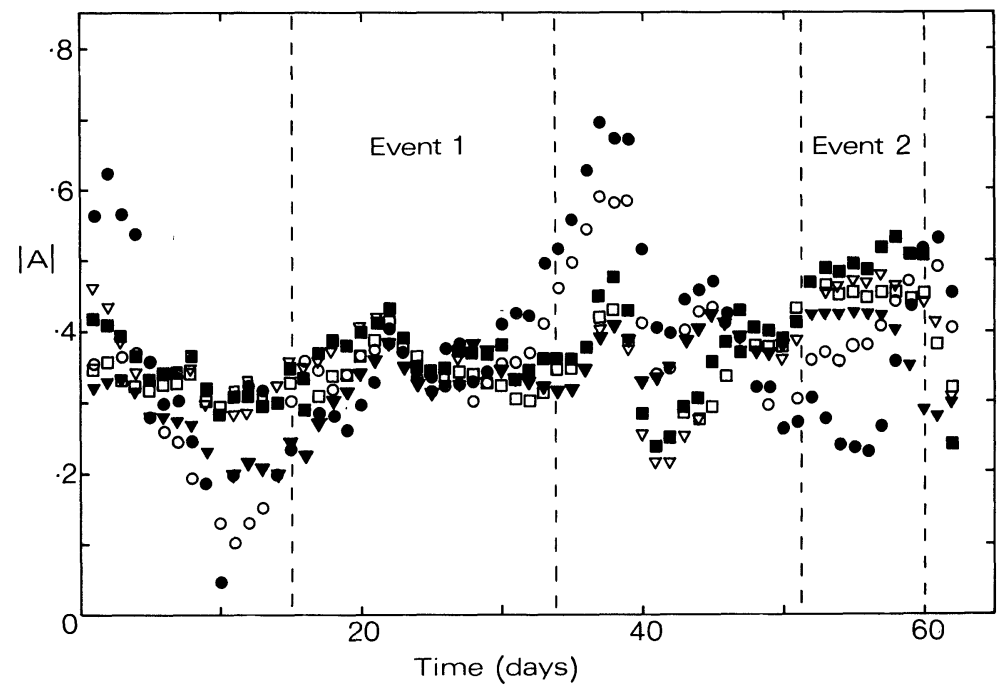

Fig. 6. Time variations of the response function $|A(\omega)|$ for the observatories ESK (๑), STO (०), WIN ( $\boldsymbol{\square})$, WIT ( $\square)$, DBS $(\boldsymbol{\nabla})$, and NGK $(\nabla)$. 


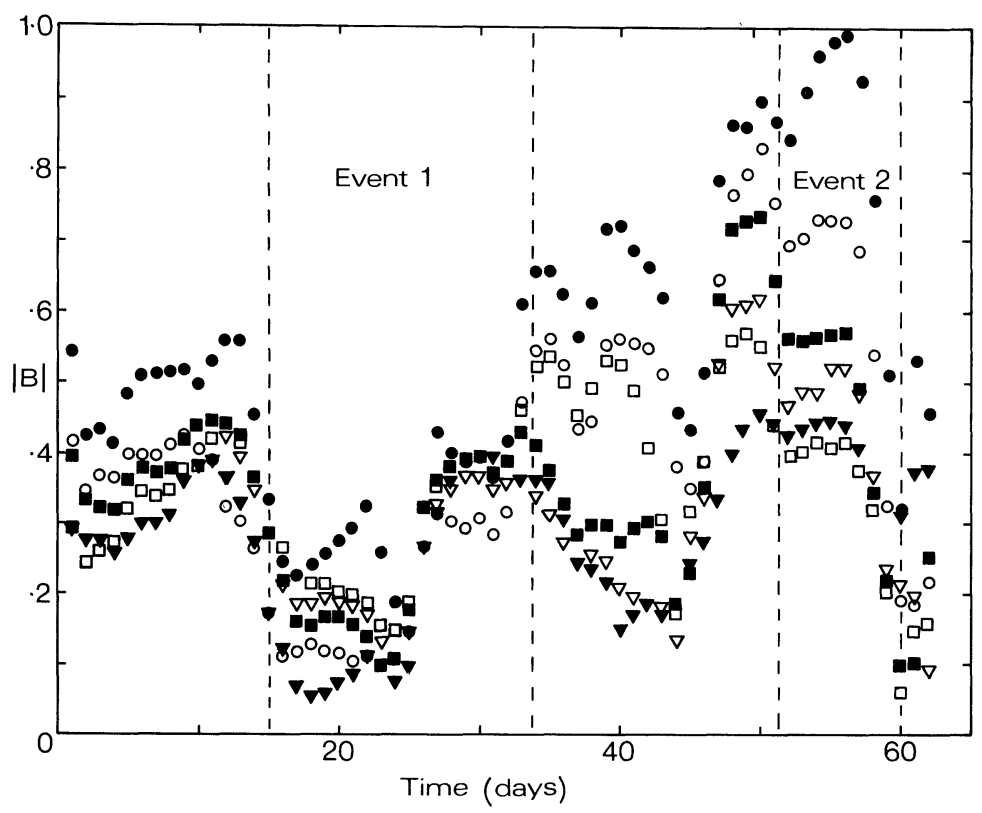

Fig. 7. Time variations of the response function $|B(\omega)|$.

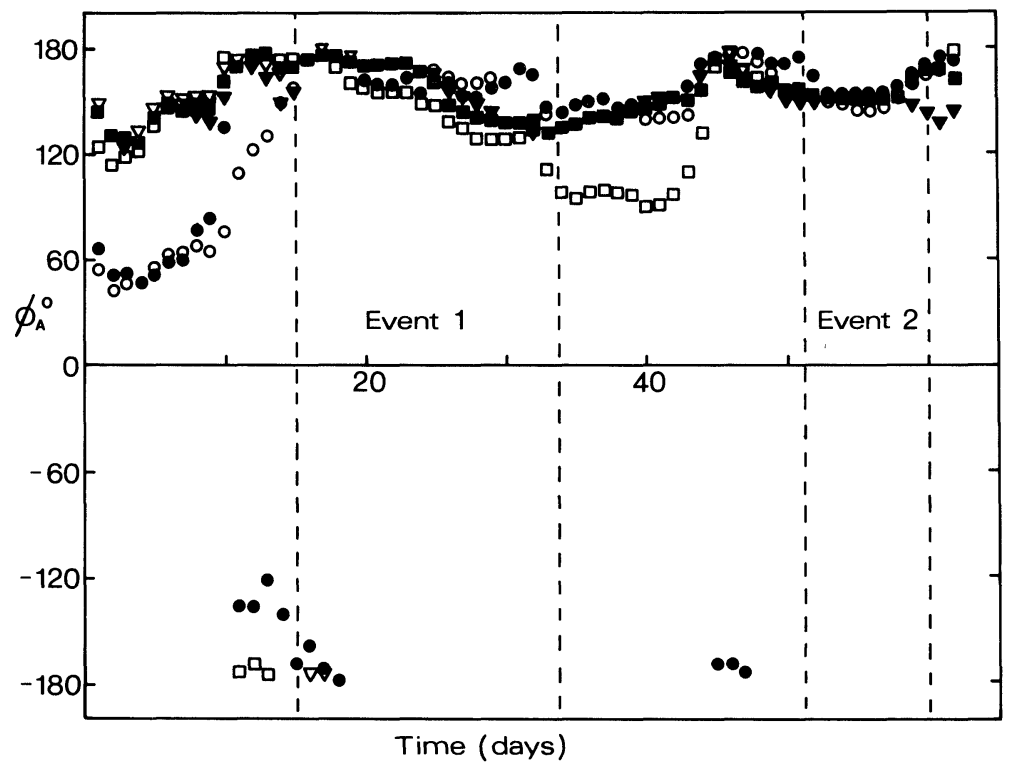

Fig. 8. Time variations of the phase of $A(\omega)$.

of $|A(\omega)|$ during event 1 . There, all the estimates, for all the observatories, lie within the range 0.3 to 0.4 ; very often the grouping is much tighter. During event 2 , on the other hand, the response is always consistent throughout the event, but the value ranges from 
0.2 to 0.5 at different observatories.

The same division of the record can be made when the response $|B(\omega)|$ is examined (Fig. 7). Event 1 shows as a distinct trough in the values of $|B(\omega)|$ at all observatories, indicating that although there is $D$ power present during the event (see Fig. 4), it is not correlated with the $Z$ power. Event 2 shows the same sort of behaviour for $B$ as for $A$ : consistent, but different responses at each station, ranging in value from 0.4 to 0.9 . The phase of the $A$ response (Fig. 8) is much less scattered than the modulus at times outside the two events. However, it is particularly consistent during the two events, with values of $150-170^{\circ}$ during event 1 , and $145-155^{\circ}$ during event 2 . It is notable that, throughout both events, not only is the phase stable with time, but its value is the same at all the observatories, even during event 2 , when the modulus differs by a factor of as much as two from one station to another.

The distinct differences in the behaviour of the response during events 1 and 2 indicate that they represent fundamentally different modes of induction. The similarity of $|A|$ at different observatories, and the low value of $|B|$, during event 1 , are compatible with induction in the deep, spherically symmetric part of the Earth by a uniform, axially symmetric source such as the ring current. In other words, $A$ is a valid estimate of the global response $G$ defined by Eq. (5). In contrast, during the course of event 2 , both $|A|$ and $|B|$ show enormous differences from one observatory to another. The source is clearly non-uniform, and the differences in the response are associated with the different locations of the observatories in relation either to the external or the internal current systems. The mode of induction is that described in Subsections 3.1 and 3.3.

The importance of the direct effect of the electrojet is indicated by the response at Eskdalemuir and Stonyhurst, the two most northern observatories. At all times, they show the largest departures from the mean of the other observatories. Furthermore, there is a clear dependence of $|B|$ on geomagnetic latitude during event 2 . The variability of $|B|$ indicates that the orientation of the current systems responsible for the $D$ variations fluctuates considerably, perhaps sufficiently to ensure that, when an average is taken over a sufficiently large body of data, the 'normal' vertical field is eliminated, and the resultant $B$ response properly reflects the current flow in the surface sheet, as most analyses assume. There is much less scatter in the $A$ response, presumably because of the more stable geometry of the east-west current systems. However, the oscillations seen at $0-5$ days, and from 35-50 days, which are particularly noticeable at the more northerly observatories, are very likely due to north-south movement of the auroral oval.

The phase of the response is particularly interesting. Two distinct phases are observed for the two events, each fairly consistent with time, and independent of the observatory. The consistency of the phase estimates at different observatories, even when the inducing field is non-uniform, is probably to be expected. Any redistribution of the current in the surface layer by conductivity anomalies will be a direct current effect, and should not alter the phase. However, the phase observed when an event excites current principally in the surface layer is bound to differ from that observed when the source is more uniform, and forces the current to flow deeper. The difference between the phases in events 1 and 2 is only $10-20^{\circ}$, but it is in the direction which would be expected. The implication of this argument is that the larger value, which may be as great as $170^{\circ}$, is the 
true phase of the global $P_{1}^{0}$ response.

This analysis shows that the response function relating the vertical and horizontal components is strongly affected by modes of induction other than that represented by a $P_{1}^{0}$ inducing field operating on a spherically symmetric conductivity structure. At times, a substantial fraction of the fields observed at geomagnetic latitudes greater than $53-54^{\circ}$ may originate in the auroral electrojet, even at periods of 2 days or more. The effects of current redistribution in the surface layer are not easy to separate from those of source geometry, although the differences in $|A|$ during event 2 are not always those to be expected from the observatory latitude. What is certain is that indiscriminate averaging will not eliminate either the electrojet or surface sheet response. However, it is perfectly possible to recognise global induction events, and when isolated they will yield more reliable and precise estimates of the global electromagnetic response.

\section{Conclusions}

In the period band 1-3 days, 'mode mixing' certainly occurs. Sometimes, ring current induction is dominant, causing the induced currents to flow primarily in the deep, spherically symmetric part of the Earth. At other times, substorm induction predominates, and a significant part of the internal current flows in the laterally heterogeneous outer shell. The response estimates obtained at such times do not correspond to the global $P_{1}^{0}$ response of the Earth, because (a) the spatial scale of the inducing field is much less than for the $P_{1}^{0}$ harmonic, and (b) the modulus of the response is determined by the location of the observatory relative to the current system, whether external or internal.

The phase of the response is much less sensitive to the mode of induction, because the redistribution of currents is essentially a direct current process at these periods, and does not alter the phase.

The conclusions have two consequences for attempts to define a better strategy for the determination of the global electromagnetic response:

(i) The results I have shown apply to a relatively long period band. At periods less than one day, the mode mixing will be more severe, because the spectrum is increasingly dominated by substorm fields, and because even the spatially uniform fields induce significant current in the outer shell. It will be correspondingly more difficult to sort out events which generate a global $P_{1}^{0}$ response. Probably the only chance of isolating this response will lie in the selection of stations which are as far as possible from current concentrations, either internal or external.

As far as internal currents are concerned, it can be anticipated that observatories at the centre of extensive blocks of homogeneous crystalline basement, flanked by highly conducting regions (seas or sedimentary basins) should meet this specification. Nonetheless, whatever might be predicted on the basis of the geology, it is always preferable to check the model by an intensive programme of geomagnetic deep sounding and magnetotelluric measurements in the vicinity of the observatory, to determine the actual distribution of current in the surface layer. In Europe, there already exists a relatively high density both of magnetic observatories, and of magnetic sounding or 
magnetotelluric observations, and it would seem a worthwhile task to compile the available data, supplementing it where necessary by new measurements. The compilation could be presented in the form of a map of the equivalent currents, which should prove extremely valuable in determining which observatories, if any, would be likely to yield worthwhile response estimates at the intermediate periods. If sufficient data were available, it might be possible to devise a means of correcting the observations for the effects of the surface layer, as Larsen has done in Hawaii (LARSEN, 1975).

The effects of the electrojet can be minimised by using only observatories with geomagnetic latitudes less than $54^{\circ}$. The results discussed in Section 4 indicate that the response at more northerly observatories may be very susceptible to fluctuations in the position of the auroral oval.

(ii) At periods of 1-10 days, mode mixing is still sufficiently important to bias the response estimates. It can be avoided by choosing those parts of the data which genuinely correspond to a $P_{1}^{0}$ inducing field, by selecting the appropriate demodulates, and incorporating them alone into the overall response estimate. The criteria for selection must be based on the spatial uniformity of the response. A possible scheme based on this approach would be:

(a) Select frequency bands, compute the demodulated time series for each band, and display the amplitude of the $H, D$, and $Z$ demodulates. Those observatories which showed poor spatial coherence in the waveforms of the demodulates would be eliminated from the computation.

(b) The $H$ demodulates of the remaining observatories are fitted to a simple spherical harmonic model, $P_{1}^{0}$ perhaps, thereby generating a new time series $H_{1}^{0}$, the amplitude of the $P_{1}^{0}$ spherical harmonic component of the horizontal field. This time series can be regarded as the best estimate of the 'input' to a system for which the $Z$ demodulates at any one observatory are the 'output'. The advantage of combining the $H$ records from different observatories is that it should eliminate events which are not spatially coherent.

(c) An electromagnetic response function is computed for each observatory in turn, between the local vertical field, $Z_{i}$, and the $P_{1}^{0}$ horizontal field:

$$
Z_{i}(\omega)=G_{i}(\omega) H_{1}^{0}(\omega)+\varepsilon_{i}(\omega) .
$$

Because $H_{1}^{0}(\omega)$ should be noise-free, the usual estimate of the response function

$$
G_{i}(\omega)=\left\langle Z_{i}(\omega) H_{1}^{0 *}(\omega)\right\rangle /\left\langle H_{1}^{0}(\omega) H_{1}^{0 *}(\omega)\right\rangle
$$

should not be biassed by auto power noise. It is preferable to compute a separate response, $G_{i}(\omega)$, for each station, rather than first combining the $Z_{i}(\omega)$ to form $Z_{1}^{0}(\omega)$, because noise levels in $Z$ at some stations are very high, and would severely bias the stacked time series.

(d) Instead of immediately computing an overall transfer function estimate, it would be preferable to first make time-local estimates, as described in Section 4. These would form the basis for discarding any remaining stretches of data which were contaminated by source or surface current effects.

(e) The response functions for individual stations, $G_{i}$, are finally combined to yield 
an overall estimate of the $P_{1}^{0}$ response:

$$
W_{1}^{0}(\omega)=Z_{1}^{0}(\omega) / H_{1}^{0}(\omega) .
$$

I should like to take this opportunity of acknowledging the very great debt I owe to the late Sir Edward Bullard, who, as my supervisor, first interested me in electromagnetic induction in the Earth, and who taught me very much of what I know about geophysics. If this paper has anything to offer, it is because I am following the ground rules for a scientific investigation which he implanted in my mind; any shortcomings are my own responsibility entirely.

\section{REFERENCES}

BaILEY, R. C., Inversion of the geomagnetic induction problem, Proc. R. Soc. Lond., A, 315, 185-194, 1970.

BANKs, R. J. Geomagnetic variations and the electrical conductivity of the upper mantle, Geophys. J. R. Astr. Soc., 17, 457-487, 1969.

BANKs, R. J., The overall conductivity distribution of the Earth, J. Geomag. Geoelectr., 24, 337-351, 1972.

Banks, R. J., Data processing and interpretation in Geomagnetic Deep Sounding, Phys. Earth Planet. Inter., 7, 339-348, 1973.

BANKs, R. J., Complex demodulation of geomagnetic data and the estimation of transfer functions, Geophys. J. R. Astr. Soc., 43, 87-101, 1975.

Banks, R. J., The use of equivalent current systems in the interpretation of Geomagnetic Deep Sounding data, Geophys. J. R. Astr. Soc., 56, 139-157, 1979.

Connerney, J. E. P. and A. F. KuCKes, Gradient analysis of geomagnetic fluctuations in the Adirondacks, $J$. Geophys. Res., 85, 2615-2624, 1980.

Ducruix, J., V. Courtillot, and J.-L. Le Mouel, The late 1960s secular variation impulse, the eleven year magnetic variation and the electrical conductivity of the deep mantle, Geophys. J. R. Astr. Soc., 61, 73-94, 1980.

Harwood, J. W. and S. R. C. Malin, Sunspot cycle influence on the geomagnetic field, Geophys. J. R. Astr. Soc., 50, 605-619, 1979.

Hughes, T. J. and G. Rostoker, A comprehensive model current system for high latitude magnetic activity; I. The steady state system, Geophys. J. R. Astr. Soc., 58, 525-569, 1979a.

Hughes, T. J. and G. Rostoker, A comprehensive model current system for high latitude magnetic activity: II. The substorm component, Geophys. J. R. Astr. Soc., 58, 571-581, 1979 b.

LAHIRI, B. N. and A. T. PRICE, Electromagnetic induction in non-uniform conductors, and the determination of the conductivity of the Earth from terrestrial magnetic variations, Philos. Trans. R. Soc. Lond., A, 237, 509-540, 1939.

LARSEN, J. C., Low frequency $(0.1-6.0 \mathrm{cpd})$ electromagnetic study of deep mantle conductivity beneath the Hawaiian Islands, Geophys. J. R. Astr. Soc., 43, 17-46, 1975.

Malin, S. R. C. and A. M. IsiKara, Annual variation of the geomagnetic field, Geophys, J.R. Astr. Soc., 47, 445-457, 1976.

Malin, S. R. C., The $S_{q}$ current system during the IGY, Geophys. J. R. Astr. Soc., 49, 515-529, 1977.

PARKER, R. L., The inverse problem of electrical conductivity in the mantle, Geophys. J. R. Astr. Soc., 22, 121-138, 1971.

PARKER, R. L., The inverse problem of electromagnetic induction: existence and construction of solutions based upon incomplete data, J. Geophys. Res., 85, 4421-4428, 1980.

Parkinson, W. D., Directions of rapid geomagnetic fluctuations, Geophys. J. R. Astr. Soc., 2, 1-13, 1959.

PRICE, A. T., The theory of magneto-telluric methods when the source field is considered, J. Geophys. Res., 67, 1907-1918, 1962.

Weidelt, P., The inverse problem of geomagnetic induction, Z. Geophysik., 38, 257-289, 1972. 